\title{
Simplified Methods for Estimation of Plasma Clearance of Iohexol in Dogs and Cats
}

\author{
Isabelle Goy-Thollot, Stéphane Besse, François Garnier, Magali Marignan, and Paul Y. Barthez
}

\begin{abstract}
The purpose of this study was to evaluate simplified methods for iohexol plasma clearance estimation in dogs and cats. Serial blood samples were taken before and 5, 20, 40, 60, 80, 100, 120, 150, 180, and 240 minutes after a bolus injection of iohexol in $51 \mathrm{dogs}$ and 25 cats. Iohexol plasma concentration was determined with X-ray fluorescence. Clearance was calculated by dividing the injected dose by the area under the plasma tracer elimination curve estimated with a 2-compartment pharmacologic model. Clearance was normalized to body surface area (BSA). The 10-point clearance was used as a reference for the evaluation of simplified methods. A 2-sample method based on a single exponential fit and a single-sample method based on a linear quadratic model were investigated. Simplified methods were evaluated by calculating the standard deviation of the difference (SDD) between the clearances obtained with the simplified methods and the 10-point reference method. All combinations of sampling times were evaluated. The best sampling times were chosen for dogs and cats as the ones yielding the lowest SDD. Linear regression analysis was performed between the reference method and the optimized simplified methods. The best combination of time for the 2-sample method was 5 and 120 minutes in dogs and 20 and 180 minutes in cats. The best time for sampling in the single-sample method was 120 minutes in dogs and 80 minutes in cats. Plasma clearance of iohexol can be estimated in dogs and cats from 1 or 2 blood samples with a reasonable margin of error.
\end{abstract}

Key words: Glomerular filtration rate; Kidney; Tracer.

$\mathbf{L}$ imited sampling strategies for plasma clearance procedures have been investigated extensively in humans, ${ }^{1-6}$ and more recently in animals, ${ }^{7-10}$ for a quick, inexpensive, and clinically acceptable estimation of glomerular filtration rate (GFR). Decreasing the number of samples from 12 to 4 in a 2-compartment pharmacologic model for ${ }^{99 \mathrm{~m}} \mathrm{Tc}$-pentetate and ${ }^{131} \mathrm{I}$ OIH plasma clearance in dogs and cats yielded only limited error on clearance estimation. ${ }^{10}$ Further decrease in the number of sample requires simplification of the pharmacologic model to a single compartment, or making assumptions regarding tracer distribution that could depend on demographic characteristics of the population (ie, species, body size, age, sex, expected clearance). Different strategies based on pharmacologic backgrounds have been used to estimate clearance on the basis of 1 or 2 samples. Most of these methods use a finite population sample to establish the relationship between clearance and tracer concentration, at given times, with the use of linear or nonlinear regression analysis, from which equation parameters and best sampling times are extracted..$^{2-5,9,11-14}$ Validation of these methods is needed to estimate the error associated with the simplified procedure and to establish an algorithm for simplified methods that can be used in practice. The larger the sample, the more reliable the simplified method will be.

From the The Département Animaux de Compagnie (GoyThollot, Marignan, Barthez) and the Département des Sciences Biologiques (Garnier, Besse), École Nationale Vétérinaire de Lyon, 1 Avenue Bourgelat-B.P. 83, 69280 Marcy l'Etoile, France. Presented at the annual ACVIM meeting in Dallas, TX, in 2002.

Reprint requests: Dr Paul Barthez, DVM, PhD, Faculteit der Diergeneeskunde, Universiteit Utrecht, P.O. Box 80.164, 3508 TD Utrecht, The Netherlands; e-mail: p.barthez@vet.uu.nl.

Submitted April 6, 2005; Revised June 14, 2005; Accepted June 30, 2005 .

Copyright $@ 2006$ by the American College of Veterinary Internal Medicine

0891-6640/06/2001-0007/\$3.00/0
The purpose of this study was to validate a 2-sample and a single-sample method for estimation of plasma clearance of iohexol in dogs and cats.

\section{Materials and Methods}

\section{Animals}

Fifty-one privately owned adult dogs and 25 cats of different breeds were included in the study. Thirty-one dogs and 19 cats were clinically healthy. Twenty dogs and 6 cats presented at the veterinary teaching hospital for evaluation of renal function. The ages of the dogs ranged from 2 months to 12.5 years (mean $\pm \mathrm{SD}$, $4.8 \pm 3.6$ years), and the ages of the cats ranged from 1 to 15.6 years $(5.2 \pm 4.4$ years). Body weight $(\mathrm{BW})$ ranged from 2.1 to $66 \mathrm{~kg}(24.1 \pm 13.6 \mathrm{~kg})$ for dogs and from 3 to $6.5 \mathrm{~kg}(4.2 \pm 1.0 \mathrm{~kg})$ for cats. All animals were well hydrated and were allowed to drink water ad libitum during the experiment.

\section{Procedure}

Catheters were placed in 2 peripheral veins, and 1 heparinized blood sample $(2 \mathrm{~mL})$ was collected. One catheter was used for injection of the tracer and the other was used for blood collection. The injected volume of iohexol ${ }^{\mathrm{a}}$ was $1 \mathrm{~mL} / \mathrm{kg}$ in dogs and $1.5 \mathrm{~mL} /$ $\mathrm{kg}$ in cats. Iohexol was administered as a bolus over a $30-60$-second period. The completion of the injection represented time 0 . The catheter was flushed and removed. Ten heparinized blood samples $(2 \mathrm{~mL})$ were obtained at $5,20,40,60,80,100,120,150,180$, and 240 minutes after injection. Blood samples were centrifuged at $3,000 \times g$ for 15 minutes; plasma was separated and then frozen at $-30^{\circ} \mathrm{C}$. Iodine concentration was measured in plasma by an X-ray fluorescence technique. ${ }^{15, \mathrm{~b}}$ Iodine concentration found in the $1 \mathrm{st}$ sample, taken before the injection of the tracer, was subtracted from iodine concentration of samples taken after the injection.

\section{Clearance Calculation}

A plasma tracer elimination curve was generated from the 10 data points. A conventional 2-compartment model was used, and the curve was fitted to a double exponential function in the form of $y=a e^{-\alpha t}+b e^{-\beta t}$ by a least squares method. ${ }^{\mathrm{c}}$ The area under the curve (AUC) was calculated from the integral of the above double exponential function between 0 and infinity: $\mathrm{AUC}=a / \alpha+b / \beta$. Iohexol clearance was calculated as the injected dose divided by 


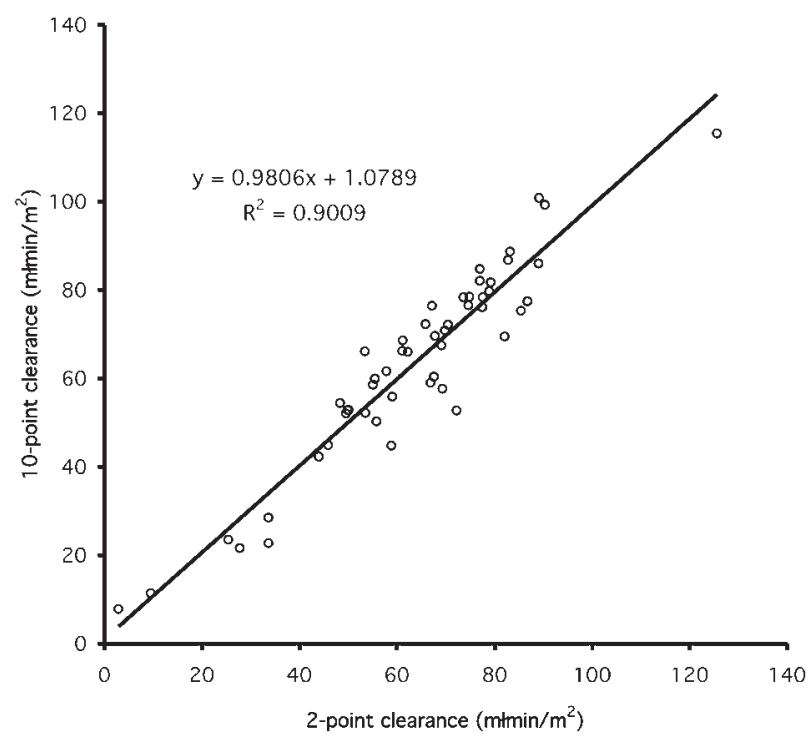

Fig 1. Linear regression analysis between 10-point clearance and 2-sample estimation (5 and 120 minutes) in 51 dogs.

AUC. ${ }^{3,16}$ Clearance values $(\mathrm{mL} / \mathrm{min})$ were normalized to body surface area (BSA; $\left.\mathrm{mL} \mathrm{min}^{-1} \mathrm{~m}^{-2}\right)$. BSA $\left(\mathrm{m}^{2}\right)$ was calculated from BW (g) by the general formula ${ }^{17}$ : BSA $=\mathrm{K} \times(\mathrm{BW})^{a} / 10^{4}$, where $\mathrm{K}$ is a shape constant (10.1 for dogs and 10.0 for cats) and $a$ is the mass exponent ( 0.71 for dogs and 0.66 for cats). ${ }^{15,17,18}$ The normalized 10-point clearance was considered a reference for the evaluation of simplified methods.

\section{Two-Sample Clearance Estimation}

The AUC was estimated by fitting 2 selected data points to a monoexponential function in the form of $C=C_{0} e^{-a t}$, where $C$ is the concentration at time $t$, and $C_{0}$ and $a$ are parameters calculated from the plasma concentrations of iohexol $C_{1}$ and $C_{2}$ at times $T_{1}$ and $T_{2}$. The AUC was calculated from $C_{0}$ and $a$ by integration of the monoexponential function from 0 to infinity: $\mathrm{AUC}=C_{0} / a$. Plasma clearance of iohexol was obtained by dividing the injected dose by AUC. Two-sample plasma clearances were calculated with all possible combinations of 2 points from the 10-point experimental data. Clearances were normalized to BSA.

\section{Single-Sample Clearance Estimation}

The volume of distribution of the tracer $V_{t}$, was calculated as the dose injected, $D$, divided by the plasma concentration, $C: V_{t}=D / C$. A nonlinear regression analysis was performed between the unnormalized 10-point plasma clearance and $V_{t}$ at a given time with a linear quadratic model in the form of Clearance $=a V_{t}^{2}+b V_{t}+c{ }^{9}$ The parameters of the regression equation were determined for dogs and cats for all sampling times. Clearances then were estimated from the linear quadratic equation and normalized to BSA.

\section{Statistical Analysis}

The mean and standard deviation of the difference (SDD) between the simplified single- and 2-sample methods, and the 10point reference clearance were determined for all combinations of sampling times for the 2-sample method and for all sampling times for the single-sample method. The combination of time yielding the smallest SDD between the 10-point reference and the simplified method was selected as the optimum. A linear regression analysis was performed between the 10-point reference method and each optimized simplified method. The concordance correlation co- efficient, Pearson correlation coefficient, and accuracy coefficient were computed for each optimized simplified method. ${ }^{19}$ Significance was set at $P<.05$.

\section{Results}

\section{Two-Sample Method}

In dogs, 10-point iohexol plasma clearance ranged from 7.8 to $115.4 \mathrm{~mL} \mathrm{~min} \mathrm{~m}^{-1}$. The smallest SDD was obtained for the combination of sampling times 5 and 120 minutes $(\mathrm{SDD}=6.99$, mean $=-0.16)$. Linear regression analysis between the 10-point reference clearance and the 2-sample clearance $(5,120)$ yielded a regression equation not significantly different from the line of identity (Fig 1) with coefficient of determination $R^{2}=.9009$. Concordance coefficient was .930 (Pearson $=.949$, accuracy coefficient $=.980$ ).

In cats, 10-point iohexol plasma clearance ranged from 6.10 to $72.85 \mathrm{~mL} \mathrm{~min}^{-1} \mathrm{~m}^{-2}$. The smallest SDD was obtained for the combination of sampling times 20 and 180 minutes $(S D D=2.42$, mean $=-1.55)$. Linear regression analysis between the 10-point reference clearance and the 2-sample clearance yielded a regression equation not significantly different from the line of identity (Fig 2) with coefficient of determination $R^{2}=$ .9700. Concordance coefficient was .939 (Pearson $=$ .985 , accuracy coefficient $=.954$ ).

\section{Single-Sample Method}

In dogs, the smallest SDD was obtained for the 120minute sampling time $(\mathrm{SDD}=9.9$, mean $=-1.1)$. Linear quadratic regression analysis between $V_{t}$ of iohexol at 120 minutes and the unnormalized 10-point clearance yielded the following regression equation $\left(R^{2}=.9473\right)$.

$$
\begin{aligned}
\text { Clearance }(120 \text { minutes })= & -0.0099 V_{t}^{2} \\
& +2.8436 V_{t}-4.9238
\end{aligned}
$$

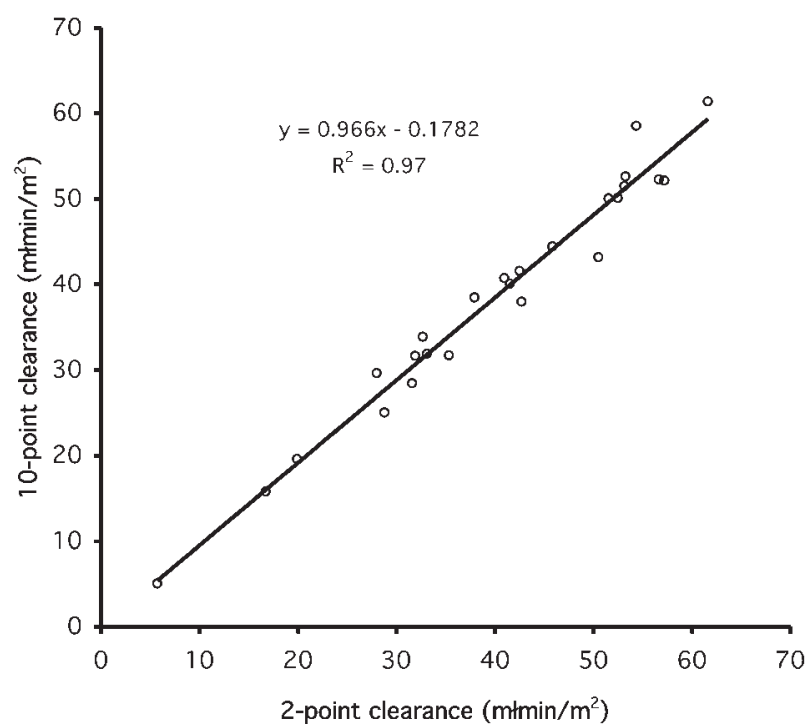

Fig 2. Linear regression analysis between 10-point clearance and 2-sample estimation (20 and 180 minutes) in 25 cats. 


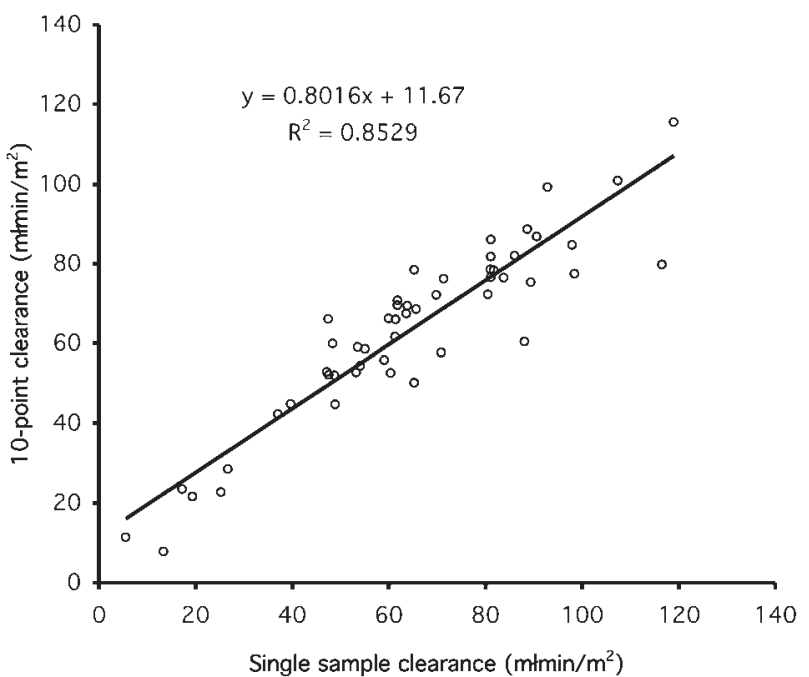

Fig 3. Linear regression analysis between 10-point clearance and single-sample estimation (120 minutes) in 51 dogs.

Linear regression analysis between the 10-point reference clearance and the estimated single sample clearance (120 minutes) yielded a regression equation significantly different from the line of identity with coefficient of determination $R^{2}=.8529$ (Fig 3). Concordance coefficient was .895 (Pearson $=.925$, accuracy coefficient $=.970$ ).

In cats, the smallest SDD was obtained for the 80minute sampling time $(\mathrm{SDD}=4.17$, mean $=-0.3$ ). Linear quadratic regression between $V_{t}$ of iohexol at 80 minutes and the unnormalized 10-point clearance yielded the following regression equation $\left(R^{2}=.9487\right)$.

Clearance $(80$ minutes $)=-0.3088 V_{t}^{2}+5.833 V_{t}-2.4825$

Linear regression analysis between the 10-point reference clearance and the estimated single-sample clearance ( 80 minutes) yielded a regression equation not significantly different from the line of identity with coefficient of determination $R^{2}=.9378$ (Fig 4). Concordance coefficient was .930 (Pearson $=.968$, accuracy coefficient $=.960$ ).

\section{Discussion}

The results of this study confirm that plasma clearance of a glomerular tracer can be estimated by both 2- and single-sample methods, with an acceptable margin of error, compared with plasma clearance calculated with a single injection, 2-compartment method in dogs and cats. The results presented herein are in agreement with previous studies in dogs and cats, with the same or a different tracer and support of the use of a simplified plasma clearance method for routine glomerular filtration rate (GFR) estimation in clinical practice., ${ }^{9,20,21}$ Normalization was performed before calculating error associated with simplified methods, because normalized clearances are used in clinical practice. Unnormalized clearances have a larger range than normalized clearances with a potential positive effect on regression analysis and calculation of coefficient of determination. Normalization to BSA was

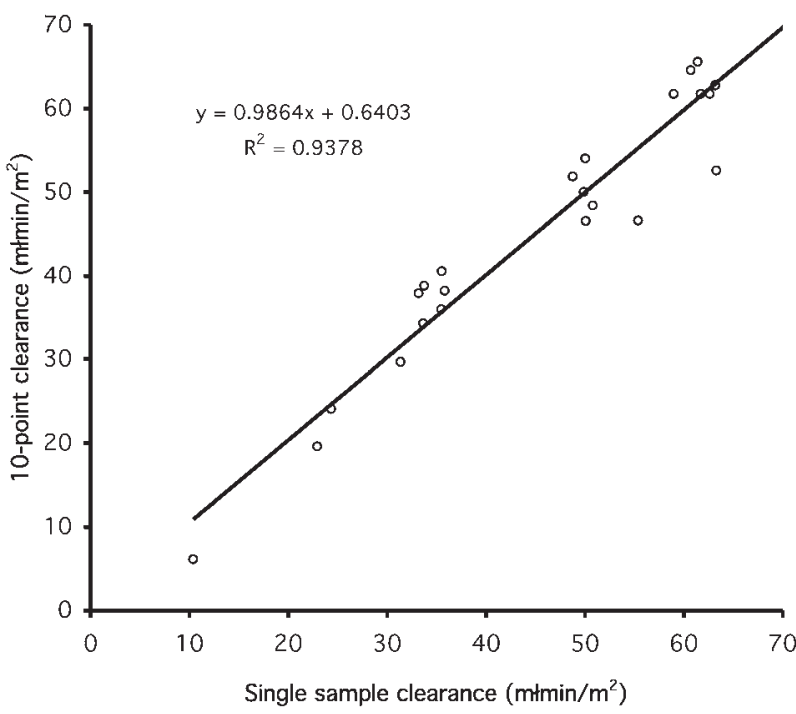

Fig 4. Linear regression analysis between 10 -point clearance and single-sample estimation ( 80 minutes) in 25 cats.

used because it has been shown to be the optimal method of normalization in dogs. ${ }^{15}$

Iohexol is an iodinated, water-soluble, nonionic, lowosmolar contrast medium that has been shown to be a good alternative to inulin and radioactive tracers for estimation of GFR in human, ${ }^{22,23}$ pig, ${ }^{24}$ horse, ${ }^{8}$ $\operatorname{dog}{ }^{25-27}$ and cat. ${ }^{28,29}$ Advantages of iohexol over other tracers include lack of radiation hazard and associated radiation protection measures, its availability, the ease of measuring plasma concentration, and the possibility of remote and delayed assay. ${ }^{30}$ Iodine is stable in plasma, allowing samples to be frozen for several weeks or sent by mail, which could simplify procedures and might be attractive for small-animal practitioners, who can send serum samples to a reference laboratory. ${ }^{30}$ In this study, X-ray fluorescence was chosen for the ease of measuring plasma iodine concentration with a commercially available analyzer at a relatively low cost. ${ }^{15}$ The amount of iodine injected was determined by the limit of detectability of iodine in plasma with X-ray fluorescence, and was estimated so that iodine could be measured in normal animals for the duration of the clearance procedure (4 hours). A relatively high concentration of iodine $(300 \mathrm{mg} \mathrm{I} / \mathrm{mL})$ was used to reduce the volume to be injected.

Disadvantages of iohexol over other tracers might include a large injection volume in large dogs and potential nephrotoxic effects of the contrast medium. ${ }^{22,26,27,31}$ Although acute nephrotoxicity of watersoluble iodinated contrast media has been reported in humans, several studies failed to demonstrate contrast medium-induced acute renal failure in animal models. ${ }^{32,33}$ In addition, there has been evidence of fewer adverse effects of nonionic low-osmolar contrast media, including iohexol, over ionic, high osmolar contrast media. ${ }^{34}$ Acute effects of low-osmolar contrast media on renal function have not been demonstrated in humans, ${ }^{22,35}$ and iohexol plasma clearance has been 
considered a safe procedure even in high-risk patients. ${ }^{31,36,37}$ Adverse effects after IV administration of iodinated contrast media have been reported rarely in dogs and cats, suggesting an even lower incidence of adverse reaction to IV injection of iohexol in these species. No adverse reaction was observed in this study and in other studies in dogs and cats using iohexol for GFR estimation. ${ }^{20,25-27,38}$ Moreover, no effect of consecutive iohexol injections on GFR was identified in dogs. ${ }^{25}$

Iohexol distribution space in the body is the extracellular volume and can be described with a 2compartment pharmacokinetic model, including the plasma as the 1st compartment and the interstitial space as the 2nd compartment. ${ }^{3,16,23}$ Its protein binding is negligible $(<1 \%)$ and almost $100 \%$ is recovered unmetabolized in the urine 24 hours after an IV injection. ${ }^{22}$ A conventional 2-compartment pharmacokinetic model was chosen as the reference method in this study for calculation of the area under the curve, because it describes accurately the distribution of contrast medium in the body. ${ }^{3,16}$

The use of 10 samples to estimate plasma clearance of a tracer and a 2-compartment model is an accurate method when performing curve fitting with nonlinear regression analysis, but it is time-consuming and expensive and could be stressful for debilitated patients. Efforts to reduce the number of samples is justified by both cost effectiveness and patient well-being. The minimum number of samples required to calculate plasma clearance with a 2-compartment pharmacologic model is 4 , because 4 unknown parameters must fit in the double exponential equation. ${ }^{10}$ Below 4 samples, assumptions have to be made regarding the volumes of distribution and the exchange rate of the tracer between the 2 compartments. Regression equation and appropriate sampling times must be empirically determined, resulting in greater error in clearance estimation. ${ }^{9}$

The 2-sample method presented herein was based on monoexponential estimation of the AUC with appropriate sampling times. ${ }^{9}$ A 2-compartment pharmacologic model still was assumed, but the error associated with the use of a single exponential function to fit the data points was minimized by use of a selected combination of sampling times. Another possibility to minimize error when using 2 samples is to 1st calculate clearance by use of the AUC corresponding to the late monoexponential part of the time-concentration curve, and to correct the calculated clearance with an empirically established equation, to take into account, the early phase..$^{5,8,9,20}$ Both methods have been validated, but the method used in this study has been shown to yield less error in clearance estimation. ${ }^{9}$ The difference in the best combination of sampling times for dogs (5 and 120 minutes) and cats (20 and 180 minutes) found in this study probably reflects differences in the population and mean clearance. Best combination of sampling times for the same method, but using ${ }^{99 \mathrm{~m}} \mathrm{Tc}$-pentetate in a group of $21 \mathrm{dogs}$ and 18 cats, was 20 and 180 minutes in both dogs and cats. ${ }^{9}$
Single-sample methods of clearance estimation are based on a nonlinear relationship between clearance and volume of distribution at a given time..$^{4,9,14,39}$ The error associated with the use of a single-sample method was greater than with a 2-sample method, and the benefit of reducing the number of samples from 2 to 1 might not compensate for the increase in error. The linear quadratic equation has been shown to give a better estimate of GFR, ${ }^{9}$ and the optimal sampling time has been shown in humans to be dependent of clearance itself. $^{40}$ In a similar study that used ${ }^{99 \mathrm{~m}}$ Tc-pentetate, ${ }^{9}$ optimal sampling times have been shown to be different in dogs (120 minutes) and cats (90 minutes), which closely matches the results of this study (dogs, 120 minutes; cats, 80 minutes).

\section{Footnotes}

\footnotetext{
${ }^{\text {a Omnipaque }} 300$ (300 mg I/mL), Nycomed Imaging AS, Oslo, Norway

${ }^{\mathrm{b}}$ Oxford Lab-X-3000, Oxford Instrument Sarl, Orsay, France

${ }^{\mathrm{c}}$ Excel $2000{ }^{\circledR}$ Microsoft Corporation, Redmond, WA
}

\section{Acknowledgment}

Supported by a grant from Novartis and Hills.

\section{References}

1. Russell CD. Optimum sample times for single-injection, multisample renal clearance methods. J Nucl Med 1993;34: 1761-1765.

2. Russell CD, Dubovsky EV. Error analysis of one sample clearance methods. Eur J Nucl Med 1992;19:824-825.

3. Lear JL, Feyerabend A, Gregory C. Two-compartment, twosample technique for accurate estimation of effective renal plasma flow: Theoretical development and comparison with other methods. Radiology 1989;172:431-436.

4. Christensen AB, Groth S. Determination of 99mTc-DTPA clearance by a single plasma sample method. Clin Physiol 1986;6:579-588.

5. Brøchner-Mortensen J. A simple method for the determination of glomerular filtration rate. Scand J Clin Lab Invest 1972;30:271-274.

6. Tauxe WN, Maher FT, Taylor WF. Effective renal plasma flow: Estimation from theoretical volumes of distribution of intravenously injected 131-I orthoiodohippurate. Mayo Clin Proc 1971;46:524-531.

7. Watson AD, Lefebvre HP, Concordet D, et al. Plasma exogenous creatinine clearance test in dogs: Comparison with other methods and proposed limited sampling strategy. J Vet Intern Med 2002;16:22-33.

8. Gonda KC, Wilcke JR, Crisman MV, et al. Evaluation of iohexol clearance used to estimate glomerular filtration rate in clinically normal foals. Am J Vet Res 2003;64:14861490

9. Barthez PY, Chew DJ, DiBartola SP. Simplified methods for estimation of $99 \mathrm{mTc}$-pentetate and 131I-orthoiodohippurate (OIH) plasma clearance in dogs and cats. J Vet Intern Med 2001;15:200-208. 
10. Barthez PY, Chew DJ, DiBartola SP. Effect of sample number and time on determination of plasma clearance of technetium Tc $99 \mathrm{~m}$ pentetate and orthoiodohippurate I 131 in dogs and cats. Am J Vet Res 2000;61:280-285.

11. Tepe PG, Tauxe WN, Bagchi A, et al. Comparison of measurement of glomerular filtration rate by single sample, plasma disappearance slope/intercept and other methods. Eur J Nucl Med 1987;13:28-31.

12. Ginjaume $\mathrm{M}$, Casey $\mathrm{M}$, Barker $\mathrm{F}$, et al. A comparison between four simple methods for measuring glomerular filtration rate using technetium-99m DTPA. Clin Nucl Med 1986;11: 647-650.

13. Jacobsson L. A method for the calculation of renal clearance based on a single plasma sample. Clin Physiol 1983;3:297-305.

14. Tauxe WN, Dubovsky EV, Kidd T Jr, et al. New formulas for the calculation of effective renal plasma flow. Eur J Nucl Med 1982;7:51-54.

15. Goy-Thollot I, Chafotte C, Besse S, et al. Iohexol plasma clearance in healthy dogs and cats. Vet Radiol Ultrasound 2005. In press.

16. Sapirstein LA, Vidt DG, Mandel MJ, et al. Volumes of distribution and clearances of intravenously injected creatinine in the dog. Am J Physiol 1955;13:330-336.

17. Price GS, Frazier DL. Use of body surface area (BSA)based dosages to calculate chemotherapeutic drug dose in dogs: I. Potential problems with current BSA formulae. J Vet Intern Med 1998;12:267-271.

18. Cowgill GR, Drabkin DL. Determination of a formula for the surface area of the dog together with a consideration of formulae available for other species. Am J Physiol 1927;81:36-61.

19. Lin LI. A concordance correlation coefficient to evaluate reproducibility. Biometrics 1989;45:255-268.

20. Heiene R, Moe L. The relationship between some plasma clearance methods for estimation of glomerular filtration rate in dogs with pyometra. J Vet Intern Med 1999;13:587-596.

21. Gleadhill A, Peters AM, Michell AR. A simple method for measuring glomerular filtration rate in dogs. Res Vet Sci 1995;59:118-123.

22. Effersoe H, Rosenkilde P, Groth S, et al. Measurement of renal function with iohexol. A comparison of iohexol, $99 \mathrm{mTc}-$ DTPA, and 51Cr-EDTA clearance. Invest Radiol 1990;25:778-782.

23. Frennby B, Sterner G. Contrast media as markers of GFR. Eur Radiol 2002;12:475-484.

24. Frennby B, Sterner G, Almen T, et al. Clearance of iohexol, chromium-51-ethylenediaminetetraacetic acid, and creatinine for determining the glomerular filtration rate in pigs with normal renal function: Comparison of different clearance techniques. Acad Radiol 1996:3:651-659.
25. Finco DR, Braselton WE, Cooper TA. Relationship between plasma iohexol clearance and urinary exogenous creatinine clearance in dogs. J Vet Intern Med 2001;15:368-373.

26. Brown SA, Finco DR, Boudinot FD, et al. Evaluation of a single injection method, using iohexol, for estimating glomerular filtration rate in cats and dogs. Am J Vet Res 1996;57:105-110.

27. Gleadhill A, Michell AR. Evaluation of iohexol as a marker for the clinical measurement of glomerular filtration rate in dogs. Res Vet Sci 1996;60:117-121.

28. Miyamoto K. Clinical application of plasma clearance of iohexol on feline patients. J Feline Med Surg 2001;3:143-147.

29. Miyamoto K. Use of plasma clearance of iohexol for estimating glomerular filtration rate in cats. Am $\mathrm{J}$ Vet Res 2001;62:572-575.

30. Heiene R, Moe L. Pharmacokinetic aspects of measurement of glomerular filtration rate in the dog: A review. J Vet Intern Med 1998;12:401-414.

31. Kasiske BL, Keane WF. Laboratory assessment of renal disease: Clearance, urinalysis and renal biopsy. In: Brenner BM, ed. The Kidney. Philadelphia, PA: WB Saunders; 1996:1137-1174.

32. Katzberg RW. What do we really know about contrast medium-induced acute renal failure? Invest Radiol 1989;24: 219-220.

33. Vaamonde CA, Bier RT, Papendick R, et al. Acute and chronic renal effects of radiocontrast in diabetic rats. Role of anesthesia and risk factors. Invest Radiol 1989;24:206-218.

34. Nygren A, Ulfendahl HR. Effects of high- and low-osmolar contrast media on renal plasma flow and glomerular filtration rate in euvolaemic and dehydrated rats. A comparison between ioxithalamate, iopamidol, iohexol and ioxaglate. Acta Radiol 1989;30:383-389.

35. Cramer BC, Parfrey PS, Hutchinson TA, et al. Renal function following infusion of radiologic contrast material. A prospective controlled study. Arch Intern Med 1985;145:87-89.

36. Stake G, Monn E, Rootwelt K, et al. Influence of urography on renal function in children. A double blind study with metrizoate and iohexol. Acta Radiol 1989;30:643-646.

37. Sterner G, Frennby B, Hultberg B, et al. Iohexol clearance for GFR-determination in renal failure-Single or multiple plasma sampling? Nephrol Dial Transplant 1996;11:521-525.

38. Moe L, Heiene R. Estimation of glomerular filtration rate in dogs with 99M-Tc-DTPA and iohexol. Res Vet Sci 1995;58:138-143.

39. Balachandran S, Toguri AG, Petrusick TW, et al. Comparative evaluation of quantitative glomerular filtration rate measured by isotopic and nonisotopic methods. Clin Nucl Med 1981;6: $150-153$.

40. Tauxe WN, Bagchi A, Tepe PG, et al. Single-sample method for the estimation of glomerular filtration rate in children. $\mathrm{J}$ Nucl Med 1987;28:366-371. 